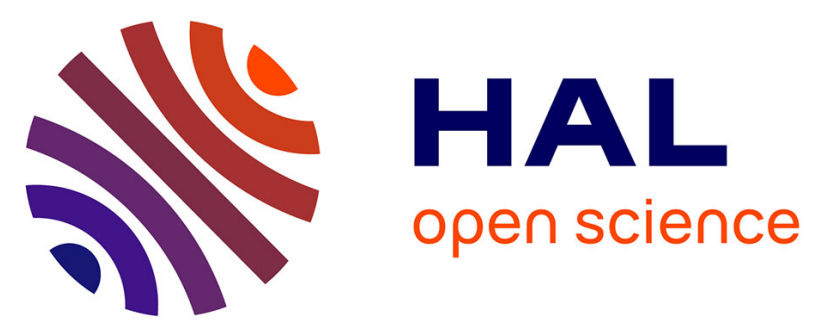

\title{
Optimized Sample Preparation and Data Processing of Data-Independent Acquisition Methods for the Robust Quantification of Trace-Level Host Cell Protein Impurities in Antibody Drug Products
}

Nicolas Pythoud, Joanna Bons, Geoffroy Mijola, Alain Beck, Sarah Cianférani, Christine Carapito

\section{To cite this version:}

Nicolas Pythoud, Joanna Bons, Geoffroy Mijola, Alain Beck, Sarah Cianférani, et al.. Optimized Sample Preparation and Data Processing of Data-Independent Acquisition Methods for the Robust Quantification of Trace-Level Host Cell Protein Impurities in Antibody Drug Products. Journal of Proteome Research, 2020, 10.1021/acs.jproteome.0c00664 . hal-02965173

\author{
HAL Id: hal-02965173 \\ https://hal.science/hal-02965173
}

Submitted on 13 Oct 2020

HAL is a multi-disciplinary open access archive for the deposit and dissemination of scientific research documents, whether they are published or not. The documents may come from teaching and research institutions in France or abroad, or from public or private research centers.
L'archive ouverte pluridisciplinaire HAL, est destinée au dépôt et à la diffusion de documents scientifiques de niveau recherche, publiés ou non, émanant des établissements d'enseignement et de recherche français ou étrangers, des laboratoires publics ou privés. 
Optimized sample preparation, data processing of data-independent acquisition methods for the robust quantification of trace-level host cell protein impurities in antibody Drug Products

\section{Authorship}

Nicolas Pythoud ${ }^{1} \ddagger$, Joanna Bons ${ }^{1} \ddagger$, Geoffroy Mijola ${ }^{2}$, Alain Beck ${ }^{2}$, Sarah Cianférani ${ }^{1}$, Christine Carapito $^{1 *}$

${ }^{1}$ Laboratoire de Spectrométrie de Masse BioOrganique, Université de Strasbourg, CNRS, IPHC, UMR7178, F-67087 Strasbourg, France.

${ }^{2} I R P F$, Centre d'Immunologie Pierre-Fabre (CIPF), F-74160 Saint-Julien-en-Genevois, France.

*Corresponding author: Christine Carapito, Laboratoire de Spectrométrie de Masse BioOrganique, Université de Strasbourg, CNRS, IPHC, UMR7178, 25 Rue Becquerel, F-67087 Strasbourg, France. ccarapito@unistra.fr

¥These authors contributed equally to this work. 


\section{Abstract}

Host cell proteins (HCPs) are a major class of bioprocess-related impurities generated by the host organism and are generally present at low levels in purified biopharmaceutical products. The monitoring of these impurities is identified as an important critical quality attribute of $\mathrm{mAb}$ formulations due to the potential risk for the product stability and efficacy but also concerns linked to the immunogenicity of some of them. While overall HCP levels are usually monitored by ELISA, mass spectrometry (MS)-based approaches are emerging as powerful and promising alternatives providing qualitative and quantitative information. However, a major challenge for LC-MS-based methods is to deal with the wide dynamic range of Drug Products and the extreme sensitivity required to detect trace-level HCPs. In this study, we developed powerful and reproducible MS-based analytical workflows coupling optimized and efficient sample preparations, library-free DIA acquisition method and stringent validation criteria. The performances of several preparation protocols and DIA versus classical DDA were evaluated using a series of four commercially available Drug Products. Depending on the selected protocols, the user has access to different information: on the one hand, a deep profiling of tens of identified HCPs, and on the other hand an accurate and reproducible $(\mathrm{CV}<12 \%)$ quantification of major HCPs. Overall, a final global HCP amount of a few tens of ng/mg mAb in these mAb samples was measured, while reaching a sensitivity down to the sub-ng/mg mAb level. Thus, this straightforward and robust approach can be intended as a routine quality control for whichever Drug Products' analysis.

\section{Keywords}

Host cell proteins (HCP), Data-Independent Acquisition (DIA), Protein quantification, Sample preparation, Data validation, Therapeutic mAbs, Adalimumab, Bevacizumab, Nivolumab, Trastuzumab 


\section{Introduction}

Monoclonal antibodies (mAbs) and derivatives constitute a major class of pharmaceutical and biotechnological active substances. Over the past three decades, a plethora of FDA and EMA approved antibodies dominated the pharmaceutical armamentarium ${ }^{1}$. They represent a 166 US\$ bln market in 2019 and $74 \%$ of total biologics sales ${ }^{2}$ and increasing demand for mAbs today drives research in a wide range of therapeutic indications ${ }^{3-4}$. Recombinant therapeutic proteins production is achieved by using cell systems, from which certain process-related impurities as host cell proteins (HCPs) derive. During the downstream process, low levels of HCPs may be co-purified with the mAb molecules and be present in the final Drug Products (DPs) ${ }^{5-7}$. HCPs are a highly diverse range of proteins, with considerable differences in physical properties $^{8}$. Indeed, some of them are enzymes catalysing degradation or comparable alterations to the final product ${ }^{9-10}$, while other may threaten patient safety by inducing unwanted immune response jeopardizing the drug productefficacy ${ }^{11-13}$. Hence, removal of these HCP impurities across the process is identified as an important critical quality attribute (CQA) of mAb DPs ${ }^{14}$. Regulatory authorities request that HCP amounts in the final Drug Product should be minimized and well controlled but without specifying precis limits ${ }^{15}$. The level of acceptable residual HCPs is reviewed on a case-by-case based on process purification capability and risk assessment including parameters such as maximum dose ( $\mathrm{mg}$ biologics/kg body weight), route of administration (intravenous or subcutaneous), frequency of dosing (acute or chronic) as well as pre-clinical and clinical data $^{16}$. Many biopharmaceutical companies are using the range of $1-100 \mathrm{ng} / \mathrm{mg} \mathrm{mAb}$ as a target for process development and for setting HCP specifications in the final DP ${ }^{3,16-18}$. The Enzyme-Linked Immunosorbent Assays (ELISA) technique is the current gold-standard for the monitoring, detection and measurement of total HCP concentration during mAb bioprocessing and in final $\mathrm{DP}^{19}$, but it suffers from several limitations: an incomplete coverage and a global quantification without relative abundance of individual $\mathrm{HCPs}^{8,16,20-23}$. 
In this context, a move towards alternative analytical methods for more accurate quantification and characterization of HCPs is needful. Mass spectrometry (MS) approaches have recently emerged as the leading proteomic analytical technology to characterize HCP contents with high confidence allowing unbiased quantification and individual HCP monitoring. While some LC-MS studies have used targeted proteomics (SRM, PRM) $)^{24-27}$ or data-dependent acquisition (DDA $)^{28-30}$, data-independent acquisition (DIA) represents a potentially superior alternative ${ }^{12,24,27,31-34}$. The key advantage of DIA modes operated on high resolution/accurate mass (HR/AM) instruments is the collection of MS2 information for all detectable species allowing the extraction of quantitative information from whole complex proteome maps ${ }^{35}$. However, 'peptide-centric' DIA data analysis requires a comprehensive spectral library ${ }^{36-37}$ to be built from DDA experiments. Besides slowing down the overall analytical process, its generation requires having access to the null cell line and/or intermediate fractions collected during the mAb bioprocess in order to obtain a comprehensive enough spectral library. Fortunately, a series of innovative algorithms have been recently introduced allowing a more straightforward application of DIA methods without requiring a spectral library ${ }^{38-41}$. Nevertheless, a bottleneck remains in MSbased quantification, when dealing with samples presenting wide dynamic range and requiring extreme sensitivity: the interference phenomenon. Previous studies attempted to overcome this limitation, caused by the overwhelming presence of biopharmaceutical peptides, by depleting the therapeutic proteins $s^{6-7,} 42-44$ or using multi-dimensional chromatography ${ }^{25,}{ }^{32-33}$. However, stringent validation criteria are still missing to ensure accurate and reproducible HCP quantification.

The focus of this study was to evaluate the different steps of the MS-based analytical workflow to improve the monitoring and global profiling of HCP impurities in a series of commercial DPs, namely adalimumab, bevacizumab, nivolumab and trastuzumab. After having finely optimized validation filters in order to obtain highly confident results for HCP monitoring, we evaluated the performances of four different sample preparations, an optimized gel fractionation, two adapted protocols from the native digestion of Huang et $a l .{ }^{43}$, and an optimized liquid digestion, to combine performant HCP content characterization and profiling to a fast and reproducible workflow. In the same time, we benchmarked 
the performances of DDA and spectral library-free DIA acquisition modes for proteins identification and quantification. In this work, the number of quantified HCP peptides and protein groups, the global label-free HCP amount, and quantification accuracy and reproductibility were assessed for benchmarking. 


\section{Experimental part}

\section{Reagents and Materials}

Adalimumab, bevacizumab, nivolumab and trastuzumab were obtained as European Union pharmaceutical-grade Drug Products from their respective manufacturers.

\section{HCP-ELISA}

ELISA assays to quantify HCPs were conducted following manufacturer's recommendation, as detailed in Supporting Information.

\section{Optimized liquid digestion protocol}

Three aliquots containing $5 \mu \mathrm{g} \mathrm{mAb}(1 \mu \mathrm{g} / \mu \mathrm{L})$ were supplemented with $45 \mu \mathrm{L}$ of $25 \mathrm{mM}$ Tris- $\mathrm{HCl}, \mathrm{pH} 8$, and $150 \mu \mathrm{L}$ of Rapid Digestion Buffer (Promega, Madison, WI, USA). Proteins were reduced with $4 \mu \mathrm{L}$ of $100 \mathrm{mM}$ TCEP for $45 \mathrm{~min}$ at $37^{\circ} \mathrm{C}$, and alkylated with $10 \mu \mathrm{L}$ of $100 \mathrm{mM}$ iodoacetamide for $1 \mathrm{~h}$ at room temperature. Proteins were digested for $1 \mathrm{~h}$ at $70^{\circ} \mathrm{C}$ using a solution of rapid Trypsin/Lys-C enzymes (Promega) at a 1:2.5 (w/w) enzymes-proteins ratio. Digestion was stopped by adding $2.2 \mu \mathrm{L}$ of formic acid (FA) and a C18 SPE (Sep-Pak C18 50 mg; Waters, Milford, MA, USA) was performed. The phase was conditioned and equilibrated with two steps with $1 \mathrm{~mL}$ methanol, three with $1 \mathrm{~mL}$ acetonitrile (ACN) and three with $1 \mathrm{~mL} 0.1 \% \mathrm{FA}$, After sample loading, the phase was washed with two steps of 1 $\mathrm{mL} 0.1 \% \mathrm{FA}$, and peptides were finally eluted with $600 \mu \mathrm{L} 60 \% \mathrm{ACN}, 0.1 \% \mathrm{FA}$.

\section{Native digestion protocol}

This protocol was adapted from Huang et $a l .{ }^{43}$. Three aliquots containing $5 \mu \mathrm{g} \mathrm{mAb}(1 \mu \mathrm{g} / \mu \mathrm{L})$ were supplemented with $5 \mu \mathrm{L}$ of $1 \mathrm{M}$ Tris- $\mathrm{HCl}, \mathrm{pH} 8$, and $185 \mu \mathrm{L} \mathrm{H} \mathrm{H}_{2} \mathrm{O}$. Proteins were digested overnight at $37^{\circ} \mathrm{C}$ using a solution of Trypsin/Lys-C enzymes (Promega) at a 1:400 enzymes-proteins ratio. Digests 
were reduced with $2 \mu \mathrm{L}$ of $303 \mathrm{mM}$ DTT for $10 \mathrm{~min}$ at $90^{\circ} \mathrm{C}$, centrifuged at 13,000 $\mathrm{g}$ for $2 \mathrm{~min}$, and the supernatants were finally acidified with $0.5 \mu \mathrm{L} F$.

\section{Rapid native digestion protocol}

Three aliquots containing $5 \mu \mathrm{g} \mathrm{mAb}(1 \mu \mathrm{g} / \mu \mathrm{L})$ were supplemented with $45 \mu \mathrm{L}$ of $25 \mathrm{mM}$ Tris- $\mathrm{HCl}, \mathrm{pH}$, and $150 \mu \mathrm{L}$ of Rapid Digestion Buffer. Proteins were digested for $1 \mathrm{~h}$ at $70^{\circ} \mathrm{C}$ using a solution of rapid Trypsin/Lys-C enzymes (Promega) at a 1:100 (w/w) enzymes-proteins ratio. The next steps were similar to the previous protocol.

\section{Semi-fractionation protocol}

After denaturation at $95^{\circ} \mathrm{C}$ for $5 \mathrm{~min}$ in loading buffer (5\% SDS, $5 \% \beta$-mercaptoethanol, $0.5 \mathrm{mM}$ EDTA, 10\% glycerol, $10 \mathrm{mM}$ Tris- $\mathrm{HCl}, \mathrm{pH} 6.8,0.05 \%$ bromophenol blue), five aliquots containing $40 \mu \mathrm{g} \mathrm{mAb}$ were loaded onto a $12 \%$-acrylamide SDS-PAGE gel. The gel was fixed with $50 \%$ ethanol, $3 \%$ phosphoric acid before staining with colloidal Silver Blue. Gel bands were cut into small pieces. Gel pieces of proteins located at higher molecular weights (MW) than mAb heavy chains, gel pieces of proteins located at lower MW than mAb light chains, and gel pieces of proteins located between mAb heavy and light chains were respectively pooled together. Proteins were in-gel reduced and alkylated as detailed in Supporting Information, and finally digested overnight at $37^{\circ} \mathrm{C}$ using a solution of Trypsin/Lys-C enzymes (Promega) at a 1:25 (w/w) enzymes-proteins ratio (we assumed the presence of $1 \mu \mathrm{g}$ proteins per gel band). Peptides were extracted with $160 \mu \mathrm{L}$ of $60 \% \mathrm{ACN}, 0.1 \% \mathrm{FA}$ for $1 \mathrm{~h}$ under agitation, and then $160 \mu \mathrm{L} \mathrm{ACN}$ for $1 \mathrm{~h}$ and 30 min under agitation.

For all protocols, after vacuum drying, peptides were resolubilized in $2 \% \mathrm{ACN}, 0.1 \% \mathrm{FA}$ to obtain a final protein concentration of $0.4 \mu \mathrm{g} / \mu \mathrm{L}$. Retention time standards iRT (Biognosys, Schlieren, Switzerland) and four accurately quantified standard proteins (on-column $10 \mathrm{fmol}$ of ADH (yeast alcohol dehydrogenase, P00330), 2 fmol of PYGM (phosphorylase b, P00489), $0.5 \mathrm{fmol}$ of BSA (bovin serum albumin, P02769), and $0.2 \mathrm{fmol}$ of ENL (yeast enolase, P00924) from the MassPREP Digestion Standard Kit (Waters, Milford, MA, USA) were spiked in each sample. 


\section{LC-MS/MS analysis for sample preparation protocols' evaluation}

Data-dependent acquisition (DDA) and data-independent acquisition (DIA) analyses were performed on a NanoAcquity UPLC device (Waters) coupled to a Q-Exactive HF-X mass spectrometer (Thermo Fisher Scientific, Bremen, Germany). Peptides (400 ng) were loaded onto a Symmetry C18 precolumn ( $20 \mathrm{~mm} \times 180 \mu \mathrm{m}, 5 \mu \mathrm{m}$ diameter particles; Waters) and eluted on a Acquity UPLC BEH130 C18 column (250 mm $\times 75 \mu \mathrm{m}, 1.70 \mu \mathrm{m}$ particles; Waters). All chromatographic gradient and MS settings are given in Supporting Information.

The dataset was deposited to the ProteomeXchange Consortium via the PRIDE partner repository with the dataset identifier PXD019668 ${ }^{45}$.

\section{DDA data analysis}

DDA data was analysed with Proteome Discoverer (v.2.4; Thermo Fisher Scientific) using SEQUEST HT (Thermo Fisher Scientific) and a FASTA file containing all Critecutulus griseus entries extracted from UniProtKB/TrEMBL $(56,566$ entries, $2019 / 09 / 30)$ as well as the retention time standards, the four standard proteins, the mAb heavy and light chains and common contaminants. Trysin/P was used as digestion enzyme and one missed cleavage was allowed. Oxidation of methionine and acetylation of protein $\mathrm{N}$-term were set as variable modifications, and carbamidomethylation of cysteine as fixed modification (except for the two native digestion-based protocols). Data was extracted using peptide mass tolerance of $5 \mathrm{ppm}$ and $\mathrm{MS} / \mathrm{MS}$ mass tolerance at $0.05 \mathrm{Da}$. A false discovery rate of $1 \%$ was set at PSM and peptide levels. XIC-MS1 quantification was performed using unique peptides and the chromatographic alignment was fixed to $10 \mathrm{~min}$ and $10 \mathrm{ppm}$ between replicates.

\section{DIA data analysis}

DIA data was analysed using directDIA implemented in Spectronaut (v.13.0; Biognosys) using the following settings and the same FASTA file as described above. Trysin/P was used as digestion enzyme and one missed cleavage was allowed. Oxidation of methionine and acetylation of protein $\mathrm{N}$-term were 
set as variable modifications, and carbamidomethylation of cysteine as fixed modification (except for the two native digestion-based protocols). Data was extracted using dynamic mass tolerances. Identification was performed using $1 \%$ precursor q-value cutoff. Quantification was performed using interference correction and at least three fragment ions used per peptide. Quantity is based on MS2 XIC peak areas. Non-identified precursors in rows with at least one q-value below 0.01 were selected for iRT profiling, by enabling carrying over the average template peak position.

\section{HCP quantity estimation}

From the export, preliminary filters were applied to remove oxidized and acetylated precursors and their counterparts, precursors not inferred to host organism proteins or standard proteins, and precursors with charge states different from two and three. To this, additional pre-filters were applied for DDA analysis to remove shared, not quantified precursors, as well as precursors with rank $>1$, and with $\mid$ RT Sequest - RT Top Apex $\mid>10$ min.

After applying stringent validation filters, peptide intensity was obtained by summing all precursor intensities and protein intensity by summing the three most intense peptides. The universal signal response factor ${ }^{46}$ (MS signal $/ \mathrm{mol}$ of protein) was calculated using PYGM, and allowed estimating protein mol quantities. Using MW and injected mAb quantities, individual HCP $\mathrm{ng} / \mathrm{mg} \mathrm{mAb}$ amounts were estimated. 


\section{Results and discussion}

The different steps of the MS-based analytical workflow, sample preparation, data acquisition and data analysis, were evaluated as presented in Figure 1. We worked with a series of four different mAbs, adalimumab, bevacizumab, nivolumab, and trastuzumab. We prepared them as complete technical triplicate (technical quintuplicate for the semi-fractionation protocol) according to four different protocols: two protocols in denaturing conditions named optimized liquid digestion (OLD) protocol and semi-fractionation protocol, and two protocols adapted from a native digestion-based protocol proposed by Huang et al. ${ }^{43}$, called native digestion (ND) and optimized native digestion (OND). Each sample was injected on a Q-Exactive HF-X operating in DDA or DIA modes. After applying finely optimized stringent filters, a Top 3 quantification strategy, assuming that the signal of the three best responding peptides per mole of protein is constant within $\mathrm{CV}$ of less than $10 \%$, was applied to estimate absolute amounts of $\mathrm{HCPs}^{46}$, using PYGM as reference protein, and the other standard proteins (ADH, BSA, ENL) as controls.

\section{Improving data validation for more accurate results}

In order to improve quantification accuracy and robustness of the results, we first optimized the data analysis workflow by implementing a series of validation filters on precursors and peptides. Indeed, in addition to standard preliminary filters applied as described in the experimental part, a series of hereafter-called validation filters were sequentially evaluated and finally implemented in both DDA and DIA workflows. The first filter, named signal quality filter, consisted in removing precursors with at least two q-values above 0.01 and/or profiled per triplicate for DIA data, and those with missing values for DDA data. Taking the OLD protocol as an example, this filter allows eliminating up to $42 \%$ of low confident HCP peptides for nivolumab in DDA (Figure 2A, Figures S1 and S2 for other protocols). As a second filter, referred to as signal repeatability filter, all precursors with a CV above $20 \%$ were eliminated to ensure reproducibility. This was the most stringent filter since at least $75 \%$ of $\mathrm{HCP}$ 
peptides and $58 \%$ of HCP protein groups were filtered out, whatever the mAb and the acquisition mode, with OLD protocol (Figures 2A and 2B, Figures S1 and S2 for other protocols). Finally, a homology filter was applied to remove HCP peptides that could potentially result from mAb degradation or unspecific cleavages. To do so, identified HCP peptide sequences were aligned using BLASTp $^{47}$ (v.2.10.0+) against mAb chains sequences and significant hits were removed (hits were considered significant if the query length was above 6 , the coverage $80 \%$ and the identity $100 \%)$. This filter allowed detecting two problematic HCP peptides of two different mAbs (adalimumab and nivolumab) in DDA with OLD protocol (Figures 2A). Although the drop in numbers is not impressive with this last filter, removed peptides can represent a large part of the final global HCP amount due to the extreme overabundance of the mAbs when compared to the HCPs.

To note, for the semi-fractionation protocol, the validation filters were slightly different (Figure S3). The CV filter was hardly applicable due to the presence of same protein groups in different gel bands. That is why only the quality signal filter, applied for each precursor in each band and each replicate independently, and the homology filter were used. In other words, the semi-fractionation protocol provided HCP identification, but no quantification information.

Altogether, these results demonstrate that classical shotgun proteomic validation workflows are not adapted as such to analyse singular samples such as DPs and obtain high confidence in the identification and quantification of HCPs. To our knowledge, most developments/studies mainly focused on sample preparation and data acquisition for HCP profiling by MS, but so far not on the data analysis step. Indeed, additional validation filters are essential to ensure reproducible and robust HCP quantification in matrices as extreme as DPs in which a superabundant mAb cohabits with trace-level HCPs. In this optic, the proposed validation workflow renders the MS-based approaches routinely applicable for the highly performing and confident HCP monitoring.

\section{Evaluation of four sample preparation protocols fashioned for HCP detection in Drug Products}


In proteomic analysis, sample preparation is a crucial step to ensure reliable identification and accurate quantification of proteins, even more when dealing with exceptional or unique samples such as DPs. Hence, we have designed four different sample preparation protocols and evaluated their performances to combine HCP content characterization and profiling in a fast and reproducible workflow. The first protocol, named Optimized Liquid Digestion and abbreviated OLD is based on the direct digestion of mAb samples in their $\mathrm{pH}$-adjusted buffer with a mixture of rapid Trypsin/Lys-C enzymes followed by the clean-up of digestion peptides by a solid phase extraction step. The second protocol named Native Digestion (ND) is adapted from Huang et al. ${ }^{43}$. Indeed, the authors proposed an original approach consisting in an overnight digestion of the DS samples in their pH-adjusted buffer and in native conditions with trypsin. The underlying idea is to keep mAb molecules intact to protect them from enzymatic digestion, so that they can be further precipitated by heat treatment, while digested HCPs will remain in solution in the supernatant. Exploiting this idea, we have tested an inspired protocol based on the use of an overnight digestion with a mixture of Trypsin/Lys-C enzymes at a 1:400 (w/w) enzymes:proteins ratio. The third protocol evaluated, named Optimized Native Digestion (OND) was also adapted from Huang et al. ${ }^{43}$ and consisted in the digestion with a rapid Trypsin/Lys-C mixture at a 1:100 (w/w) enzymes:proteins ratio, for $1 \mathrm{~h}$ only. Finally, a semifractionation protocol consisting in fractionating the mAb sample on a $12 \%$ acrylamide gel, and generating three band pools (one for proteins located at higher MW than mAb heavy chains, one for proteins located at lower MW than mAb light chains, and one for proteins located between mAb heavy and light chains) was evaluated. The pools were then submitted to a classical in-gel procedure ${ }^{24}$, and proteins were digested overnight with a Trypsin/Lys-C mix.

The semi-fractionation protocol allows obtaining identification information on HCP peptides and protein groups (Figure S3 and Table S1). After applying the previously described validation filters, 53 to 141 peptides and 30 to 73 protein groups were identified among the different mAb samples. DIA allows identifying more peptides than DDA, while the number of identified protein groups was similar. 
Consequently, an average of three peptides were identified per protein group by DIA, against 1.8 by DDA, what strengthens the confidence in the identification.

Figure 3 reports the number of quantified HCP peptides and protein groups as well as the global HCP amount obtained with the OLD, ND, and OND protocols after applying the previously arrested validation workflow (Figures $\mathbf{2}$, S1 and S2 respectively, and Table S1). On average, six and three HCP protein groups were quantified in DIA and DDA respectively, representing a global HCP amount varying between $28 \pm 1 \mathrm{ng} / \mathrm{mg} \mathrm{mAb}$ and $196 \pm 19 \mathrm{ng} / \mathrm{mg} \mathrm{mAb}$ in DIA and $21 \pm 1 \mathrm{ng} / \mathrm{mg} \mathrm{mAb}$ and $269 \pm 12$ $\mathrm{ng} / \mathrm{mg} \mathrm{mAb}$ in DDA in the different mAb samples. To note, no quantification values were obtained for all mAb samples prepared with the OND protocol as well as the adalimumab sample prepared with the ND protocol, and acquired in DDA mode, while DIA succeeded in retrieving quantitative information for all cases. Up to 5, 9 and $10 \mathrm{HCPs}$ were quantified using the OND, ND and OLD protocols respectively. The benchmarking of MS strategies against release testing results from the manufacturer, provided by ELISA assays, resulted in global sub-ng/mg mAb amount, which is in line with other studies ${ }^{12,24,27}$ (Table S2). Altogether, this highlights that, in the majority of mAbs, joint use of DIA and ND or OLD protocol allows quantifying more HCP peptides and protein groups with a higher global HCP amount.

Besides identification and quantification performances, preparation time is another crucial parameter to consider when evaluating a sample preparation workflow. By looking at the duration of each protocol represented in Figure S4, the OND protocol offers the shortest preparation time compared to OLD and ND protocols, namely 2, 5 and 15 hours respectively. Indeed, the use of the rapid enzyme mixture in OND and OLD protocols allowed decreasing significantly the digestion time, rendering possible the application of the complete workflow within a single day.

Although the OND protocol allowed drastically reducing the preparation time, the number of quantified HCPs and their global amount were finally the less satisfying in DIA acquisition mode. The best compromise between quantification efficacy and preparation time was obtained for the OLD and ND protocols. On the one hand, the OLD protocol enables a reduced digestion time, on the other hand 
the ND protocol allowed efficient mAb precipitation, whatever the drug product considered (Figure S5), as described by Huang et $a . .^{43}$. Actually, maximum $3 \%$ of mAb remains in the native protocols, when compared to the OLD protocol, what thus reduces the dynamic range between $\mathrm{mAb}$ and residual HCPs.

All these results highlight the possibility to obtain different levels of information depending on the chosen workflow. On the one hand, thanks to the double fractionation (proteins by SDS-PAGE and peptides by RP-HPLC), the semi-fractionation protocol offers the deepest overview of the HCP content in DPs, although the whole workflow duration is longer. On the other hand, the OLD and ND protocols should be preferred when one wants to obtain an accurate and reproducible quantification of HCPs within a limited time, rendering them applicable for routine and daily quality control.

\section{Added value of Data-Independent Acquisition for HCP detection in Drug Products}

To obtain a deeper profiling of HCPs present in DPs, we have evaluated the benefit of using the DIA acquisition mode. In contrast to the sequential detection and analysis of the most abundant ions in DDA, DIA allows parallelizing the fragmentation of all detectable ions within a wide $\mathrm{m} / \mathrm{z}$ range regardless of intensity resulting in improved sensibility, reproducibility and quantification accuracy ${ }^{35}$. Consequently, a fine optimization of variable windows used to setup a DIA method allowed limiting potential interferences and improved the specificity of the DIA assays (Table S3). This method was benchmarked against a current standard label-free XIC-MS1 quantification strategy. For Top3 amount estimations, the universal response factor (MS signal/mol of protein) was assessed using the PYGM reference protein. Moreover, additional ADH, BSA and ENO reference proteins served as quantification controls. The PYGM/ADH ng/mg mAb amounts determined in the samples processed with the ND, OLD and OND protocols and analysed in DDA or DIA are represented in Figure 4A. All ratios were above the theoretical value of 0.2 , however the medians of the DIA ratios were slightly lower than those of the DDA ratios ( 0.34 against 0.35 , and 0.28 against 0.31 , for ND and OLD protocols respectively). To note, no ratios could be calculated for adalimumab samples prepared with the ND protocol and analysed in 
DDA, as well as all OND-DDA samples, since no ADH and/or PYGM peptides passed the above described validation filters. Moreover, the CVs calculated between technical triplicates were below $12.5 \%$, whatever the protocol and acquisition mode used (Figure 4B), with a lower median for DDA than DIA using ND protocol $(4.9 \%$ against $8.9 \%$, and $10.7 \%$ against $11.0 \%$, for ND and OLD protocols respectively). Again, $\mathrm{CV}$ values could not be determined for the same samples as previously mentioned since no HCP could have been quantified (Figure 3). It is worth noting that while the signal repeatability filter of $20 \%$ was applied, the actual CV values are far below this limit (Figure $4 \mathbf{B}$ ). In addition, Top3 quantification strategy provides an accurate quantification estimation, which is in accordance with previous studies ${ }^{24,46,48-50}$. Thus, the combination of the Top3 approach and a strict repeatability control strengthens quantification accuracy and reproducibility of MS-based approaches.

Similar minimal HCP ng/mg mAb amounts were reached by DDA and DIA, but not in the same mAb sample prepared with the same protocol: $0.08 \pm 0.01 \mathrm{ng} / \mathrm{mg} \mathrm{mAb}$ for adalimumab in OLD-DDA and $0.12 \pm 0.02 \mathrm{ng} / \mathrm{mg} \mathrm{mAb}$ for nivolumab in OND-DIA (no quantified HCP in the corresponding DDA sample) (Table S4). Moreover, in the study, 0.06 to 2.80 orders of magnitude were covered between the least and the most abundant HCP in DDA, and 1.11 to 2.52 orders of magnitude in DIA. This highlights that up to seven orders of magnitude between mAb and HCPs were covered whatever the acquisition mode. As already mentioned previously, concerning the samples acquired in DDA mode, no quantification results could be obtained for any mAbs prepared with the OND protocol and only three for those prepared with the ND protocol. This observation highlights that DIA generally allows improving sensitivity performance by rendering possible the extraction of signals close to the background, but unattainable with classical DDA. This points out the benefit of performing MS2-based rather than MS1-based quantification, when it comes to deal with the trace-level HCPs in DPs. Effectively, in this specific context of HCP profiling, this gain enables detecting and quantifying more HCPs, as previously discussed (Figure 3 ), and notably the less abundant HCPs present at $\mathrm{ng} / \mathrm{mg} \mathrm{mAb}$ level, among which potentially problematic protein contaminants that could have escaped to more classical monitoring assays. 
While DDA XIC-MS1-based quantification strategy provides a global profiling of the protein content but biased towards the most intense peptides/proteins and with limited accuracy, MS2-based targeted approaches $\left(\mathrm{SRM}^{51}, \mathrm{PRM}^{52-53}\right)$ afford an accurate, sensitive and reproducible quantification but with limited multiplexing. Fortunately, DIA combines the advantages of both approaches, offering an accurate, robust and complete map of the HCPs contained in the DPs ${ }^{37,54}$. In line with this, our results demonstrate that the Top3-DIA strategy is able to provide very good HCP quantification accuracy and reproducibility, while achieving a sensitivity down to the $\mathrm{ng} / \mathrm{mg} \mathrm{mAb}$ level. Moreover, it is worth noting that the DIA data analysis workflow used in this work does not require providing a spectral library, whose generation is a time- and resource-consuming step, and in fine enables to reduce the global workflow duration to the sole DPs' sample preparation, acquisition and analysis. Besides this, it is not always possible to generate a spectral library while not having access to the null cell line or to fractions highly contaminated in HCPs (as collected after harvest for instance). Hence, this spectral library-free DIA approach renders this workflow robust, ready-to-use and universally applicable for an accurate and precise HCP profiling. 


\section{Conclusion}

In summary, we have optimized a ready-to-use and universally applicable workflow for HCP monitoring using the combination of efficient sample preparation using Trypsin/Lys-C enzymes, spectral libraryfree DIA and stringent data analysis. Firstly, we have demonstrated the absolute need to apply finely optimized validation filters in order to obtain highly confident and robust results. These different filter levels allow successively eliminating low confident signals, non-reproducible results and false positive HCPs resulting eventually from mAb degradation. Then, we have compared the performances of four different sample preparations, an optimized gel fractionation, two preparation protocols adapted from the native digestion of Huang et $a l .{ }^{43}$, and an optimized liquid digestion protocol. This comparison has demonstrated that several protocols provided well-balanced performances between identification/quantification and preparation time. Actually, the semi-fractionation was optimal to obtain a deep profiling of the HCP content in DPs, but at the cost of a more time-consuming analytical workflow. The OLD and ND protocols, for their part, offer a robust and daily applicable workflow to accurately quantify HCPs with similar performances. While the use of rapid enzymes in the OLD protocol has permitted reducing the sample preparation duration to five hours only, the ND one allows eliminating the superabundant $\mathrm{mAb}$ from the sample and finally reduce the dynamic range with the trace-level HCPs. Finally, the benchmarking of spectral library-free DIA and DDA acquisition modes highlighted the promising performances of a straightforward DIA method for HCP monitoring. Indeed, it allowed increasing the quantified HCP number as well as the global abundance in most of the samples compared to classical DDA XIC-MS1 label-free quantification. Spectral library-free DIA data analysis allows obtaining a complete picture of all HCPs contained in the DPs without additional experimental requirements for spectral library generation. This straightforward and robust approach, demonstrated on various DPs, can thus be intended as a routine quality control for whichever products' analysis. 


\section{Associated content}

\section{Supporting Information}

The following supporting information is available free of charge at ACS website: http://pubs.acs.org

Supplementary Table S1: Global ng/mg mAb amounts of HCPs determined by ELISA assay.

Supplementary Table S2 (attached .xlsx file): Detailed lists of identified and quantified HCPs depending on the sample preparation protocol and the acquisition mode.

Supplementary Table S3: DIA isolation windows used for sample preparation protocols' evaluation.

Supplementary Table S4: Individual ng/mg mAb amounts range and dynamic range of HCPs depending on the sample preparation protocol and the acquisition mode.

Supplementary Figure S1: Effects of the validation filters on the number of HCP peptides and proteins in the case of native digestion protocol.

Supplementary Figure S2: Effects of the validation filters on the number of HCP peptides and proteins in the case of optimized native digestion protocol.

Supplementary Figure S3: Effects of the validation filters on the number of HCP peptides and proteins in the case of semi-fractionation protocol.

Supplementary Figure S4: Comparisons of the efficacy and the preparation time of the different sample preparation protocols in the case of DIA acquisition mode.

Supplementary Figure S5: Effect of native digestion on mAb precipitation. 


\section{Acknowledgements}

NP was financed by the Proteocardis project (ANR-15-CE14-0013-02) and JB by a fellowship from the French Ministry of Science. This study was supported by the French Proteomic Infrastructure (ProFl; ANR-10-INBS-08-03).

The dataset was deposited to the ProteomeXchange Consortium via the PRIDE partner repository with the dataset identifier PXD019668 ${ }^{45}$. 


\section{References}

1. Kaplon, H.; Muralidharan, M.; Schneider, Z.; Reichert, J. M., Antibodies to watch in 2020. MAbs 2020, $12(1), 1703531$.

2. Blockbuster Biologics 2019: Sales of Recombinant Therapeutic Antibodies \& Proteins. La Merie Publishing 2020.

3. Gilgunn, S.; El-Sabbahy, H.; Albrecht, S.; Gaikwad, M.; Corrigan, K.; Deakin, L.; Jellum, G.; Bones, J., Identification and tracking of problematic host cell proteins removed by a synthetic, highly functionalized nonwoven media in downstream bioprocessing of monoclonal antibodies. J Chromatogr A 2019, 1595, 28-38.

4. Beck, A.; Wurch, T.; Bailly, C.; Corvaia, N., Strategies and challenges for the next generation of therapeutic antibodies. Nat Rev Immunol 2010, 10 (5), 345-52.

5. Pezzini, J.; Joucla, G.; Gantier, R.; Toueille, M.; Lomenech, A. M.; Le Senechal, C.; Garbay, B.; Santarelli, X.; Cabanne, C., Antibody capture by mixed-mode chromatography: a comprehensive study from determination of optimal purification conditions to identification of contaminating host cell proteins. J Chromatogr A 2011, 1218 (45), 8197-208.

6. Joucla, G.; Le Senechal, C.; Begorre, M.; Garbay, B.; Santarelli, X.; Cabanne, C., Cation exchange versus multimodal cation exchange resins for antibody capture from $\mathrm{CHO}$ supernatants: identification of contaminating host cell proteins by mass spectrometry. J Chromatogr B Analyt Technol Biomed Life Sci 2013, 942-943, 126-33.

7. Aboulaich, N.; Chung, W. K.; Thompson, J. H.; Larkin, C.; Robbins, D.; Zhu, M., A novel approach to monitor clearance of host cell proteins associated with monoclonal antibodies. Biotechnol Prog 2014, $30(5), 1114-24$.

8. Tscheliessnig, A. L.; Konrath, J.; Bates, R.; Jungbauer, A., Host cell protein analysis in therapeutic protein bioprocessing - methods and applications. Biotechnol J 2013, 8 (6), 655-70. 
9. Kao, Y. H.; Hewitt, D. P.; Trexler-Schmidt, M.; Laird, M. W., Mechanism of antibody reduction in cell culture production processes. Biotechnol Bioeng 2010, 107 (4), 622-32.

10. Trexler-Schmidt, M.; Sargis, S.; Chiu, J.; Sze-Khoo, S.; Mun, M.; Kao, Y. H.; Laird, M. W., Identification and prevention of antibody disulfide bond reduction during cell culture manufacturing. Biotechnol Bioeng 2010, 106 (3), 452-61.

11. Goey, C. H.; Alhuthali, S.; Kontoravdi, C., Host cell protein removal from biopharmaceutical preparations: Towards the implementation of quality by design. Biotechnol Adv 2018, 36 (4), 12231237.

12. Farrell, A.; Mittermayr, S.; Morrissey, B.; Mc Loughlin, N.; Navas Iglesias, N.; Marison, I. W.; Bones, J., Quantitative host cell protein analysis using two dimensional data independent LC-MS(E). Anal Chem 2015, 87 (18), 9186-93.

13. Jawa, V.; Joubert, M. K.; Zhang, Q.; Deshpande, M.; Hapuarachchi, S.; Hall, M. P.; Flynn, G. C., Evaluating Immunogenicity Risk Due to Host Cell Protein Impurities in Antibody-Based Biotherapeutics. AAPS J 2016, 18 (6), 1439-1452.

14. Ahluwalia, D.; Dhillon, H.; Slaney, T.; Song, H.; Boux, H.; Mehta, S.; Zhang, L.; Valdez, A.; Krishnamurthy, G., Identification of a host cell protein impurity in therapeutic protein, P1. J Pharm Biomed Anal 2017, 141, 32-38.

15. U.S. Department of Health and Human Services, Food and Drug Administration; Center for Drug Evaluation and Research (CDER); Center for Biologics Evaluation and Research (CBER). Guidance for Industry Q6B Specifications: Test Procedures and Acceptance Criteria for Biotechnological/Biological Products; 1999.

16. Wang, X.; Hunter, A. K.; Mozier, N. M., Host cell proteins in biologics development: Identification, quantitation and risk assessment. Biotechnol Bioeng 2009, 103 (3), 446-58.

17. Champion, K.; Madden, H.; Dougherty, J.; Shacter, E., Defining Your Product Profile and Maintaining Control Over It, Part 2. Bioprocess Int. 2005, 5, 52-57. 
18. Eaton, L. C., Host cell contaminant protein assay development for recombinant biopharmaceuticals. Journal of Chromatography A 1995, 705 (1), 105-114.

19. Chon, J. H.; Zarbis-Papastoitsis, G., Advances in the production and downstream processing of antibodies. N Biotechnol 2011, 28 (5), 458-63.

20. Valente, K. N.; Levy, N. E.; Lee, K. H.; Lenhoff, A. M., Applications of proteomic methods for CHO host cell protein characterization in biopharmaceutical manufacturing. Curr Opin Biotechno/ 2018, $53,144-150$.

21. Bracewell, D. G.; Francis, R.; Smales, C. M., The future of host cell protein (HCP) identification during process development and manufacturing linked to a risk-based management for their control. Biotechnol Bioeng 2015, 112 (9), 1727-37.

22. Hogwood, C. E.; Bracewell, D. G.; Smales, C. M., Measurement and control of host cell proteins (HCPs) in CHO cell bioprocesses. Curr Opin Biotechnol 2014, 30, 153-60.

23. Zhu-Shimoni, J.; Yu, C.; Nishihara, J.; Wong, R. M.; Gunawan, F.; Lin, M.; Krawitz, D.; Liu, P.; Sandoval, W.; Vanderlaan, M., Host cell protein testing by ELISAs and the use of orthogonal methods. Biotechnol Bioeng 2014, 111 (12), 2367-79.

24. Husson, G.; Delangle, A.; O'Hara, J.; Cianferani, S.; Gervais, A.; Van Dorsselaer, A.; Bracewell, D.; Carapito, C., Dual Data-Independent Acquisition Approach Combining Global HCP Profiling and Absolute Quantification of Key Impurities during Bioprocess Development. Anal Chem 2018, 90 (2), $1241-1247$.

25. Doneanu, C.; Fang, J.; Alelyunas, Y.; Yu, Y. Q.; Wrona, M.; Chen, W., An HS-MRM Assay for the Quantification of Host-cell Proteins in Protein Biopharmaceuticals by Liquid Chromatography lon Mobility QTOF Mass Spectrometry. J Vis Exp 2018, 134, e55325.

26. Gao, X.; Rawal, B.; Wang, Y.; Li, X.; Wylie, D.; Liu, Y. H.; Breunig, L.; Driscoll, D.; Wang, F.; Richardson, D. D., Targeted Host Cell Protein Quantification by LC-MRM Enables Biologics Processing and Product Characterization. Anal Chem 2020, 92 (1), 1007-1015. 
27. Kreimer, S.; Gao, Y.; Ray, S.; Jin, M.; Tan, Z.; Mussa, N. A.; Tao, L.; Li, Z.; Ivanov, A. R.; Karger, B. L., Host Cell Protein Profiling by Targeted and Untargeted Analysis of Data Independent Acquisition Mass Spectrometry Data with Parallel Reaction Monitoring Verification. Anal Chem 2017, 89 (10), $5294-5302$.

28. Tait, A. S.; Hogwood, C. E.; Smales, C. M.; Bracewell, D. G., Host cell protein dynamics in the supernatant of a mAb producing CHO cell line. Biotechnol Bioeng 2012, 109 (4), 971-82.

29. Hogwood, C. E.; Tait, A. S.; Koloteva-Levine, N.; Bracewell, D. G.; Smales, C. M., The dynamics of the $\mathrm{CHO}$ host cell protein profile during clarification and protein A capture in a platform antibody purification process. Biotechnol Bioeng 2013, 110 (1), 240-51.

30. Krawitz, D. C.; Forrest, W.; Moreno, G. T.; Kittleson, J.; Champion, K. M., Proteomic studies support the use of multi-product immunoassays to monitor host cell protein impurities. Proteomics 2006, 6 (1), 94-110.

31. Zhang, Q.; Goetze, A. M.; Cui, H.; Wylie, J.; Trimble, S.; Hewig, A.; Flynn, G. C., Comprehensive tracking of host cell proteins during monoclonal antibody purifications using mass spectrometry. MAbs 2014, 6 (3), 659-70.

32. Doneanu, C. E.; Xenopoulos, A.; Fadgen, K.; Murphy, J.; Skilton, S. J.; Prentice, H.; Stapels, M.; Chen, W., Analysis of host-cell proteins in biotherapeutic proteins by comprehensive online twodimensional liquid chromatography/mass spectrometry. MAbs 2012, 4 (1), 24-44.

33. Doneanu, C. E.; Anderson, M.; Williams, B. J.; Lauber, M. A.; Chakraborty, A.; Chen, W., Enhanced Detection of Low-Abundance Host Cell Protein Impurities in High-Purity Monoclonal Antibodies Down to $1 \mathrm{ppm}$ Using Ion Mobility Mass Spectrometry Coupled with Multidimensional Liquid Chromatography. Anal Chem 2015, 87 (20), 10283-91.

34. Heissel, S.; Bunkenborg, J.; Kristiansen, M. P.; Holmbjerg, A. F.; Grimstrup, M.; Mortz, E.; Kofoed, T.; Hojrup, P., Evaluation of spectral libraries and sample preparation for DIA-LC-MS analysis of host cell proteins: A case study of a bacterially expressed recombinant biopharmaceutical protein. Protein Expr Purif 2018, 147, 69-77. 
35. Gillet, L. C.; Navarro, P.; Tate, S.; Rost, H.; Selevsek, N.; Reiter, L.; Bonner, R.; Aebersold, R., Targeted data extraction of the MS/MS spectra generated by data-independent acquisition: a new concept for consistent and accurate proteome analysis. Mol Cell Proteomics 2012, 11 (6), 0111 016717.

36. Rost, H. L.; Rosenberger, G.; Navarro, P.; Gillet, L.; Miladinovic, S. M.; Schubert, O. T.; Wolski, W.; Collins, B. C.; Malmstrom, J.; Malmstrom, L.; Aebersold, R., OpenSWATH enables automated, targeted analysis of data-independent acquisition MS data. Nat Biotechnol 2014, 32 (3), 219-23.

37. Ludwig, C.; Gillet, L.; Rosenberger, G.; Amon, S.; Collins, B. C.; Aebersold, R., Data-independent acquisition-based SWATH-MS for quantitative proteomics: a tutorial. Mol Syst Bio/ 2018, 14 (8), e8126. 38. Navarro, P.; Kuharev, J.; Gillet, L. C.; Bernhardt, O. M.; MacLean, B.; Rost, H. L.; Tate, S. A.; Tsou, C. C.; Reiter, L.; Distler, U.; Rosenberger, G.; Perez-Riverol, Y.; Nesvizhskii, A. I.; Aebersold, R.; Tenzer, S., A multicenter study benchmarks software tools for label-free proteome quantification. Nat Biotechnol 2016, 34 (11), 1130-1136.

39. Muntel, J.; Kirkpatrick, J.; Bruderer, R.; Huang, T.; Vitek, O.; Ori, A.; Reiter, L., Comparison of Protein Quantification in a Complex Background by DIA and TMT Workflows with Fixed Instrument Time. J Proteome Res 2019, 18 (3), 1340-1351.

40. Demichev, V.; Messner, C. B.; Vernardis, S. I.; Lilley, K. S.; Ralser, M., DIA-NN: neural networks and interference correction enable deep proteome coverage in high throughput. Nat Methods 2020, $17(1), 41-44$.

41. Tsou, C. C.; Avtonomov, D.; Larsen, B.; Tucholska, M.; Choi, H.; Gingras, A. C.; Nesvizhskii, A. I., DIA-Umpire: comprehensive computational framework for data-independent acquisition proteomics. Nat Methods 2015, 12 (3), 258-64, 7 p following 264.

42. Chen, I. H.; Xiao, H.; Daly, T.; Li, N., Improved Host Cell Protein Analysis in Monoclonal Antibody Products through Molecular Weight Cutoff Enrichment. Anal Chem 2020, 92 (5), 3751-3757. 
43. Huang, L.; Wang, N.; Mitchell, C. E.; Brownlee, T.; Maple, S. R.; De Felippis, M. R., A Novel Sample Preparation for Shotgun Proteomics Characterization of HCPs in Antibodies. Anal Chem 2017, 89 (10), 5436-5444.

44. Kufer, R.; Haindl, M.; Wegele, H.; Wohlrab, S., Evaluation of Peptide Fractionation and Native Digestion as Two Novel Sample Preparation Workflows to Improve HCP Characterization by LC-MS/MS. Anal Chem 2019, 91 (15), 9716-9723.

45. Deutsch, E. W.; Csordas, A.; Sun, Z.; Jarnuczak, A.; Perez-Riverol, Y.; Ternent, T.; Campbell, D. S.; Bernal-Llinares, M.; Okuda, S.; Kawano, S.; Moritz, R. L.; Carver, J. J.; Wang, M.; Ishihama, Y.; Bandeira, N.; Hermjakob, H.; Vizcaino, J. A., The ProteomeXchange consortium in 2017: supporting the cultural change in proteomics public data deposition. Nucleic Acids Res 2017, 45 (D1), D1100-D1106. 46. Silva, J. C.; Gorenstein, M. V.; Li, G. Z.; Vissers, J. P.; Geromanos, S. J., Absolute quantification of proteins by LCMSE: a virtue of parallel MS acquisition. Mol Cell Proteomics 2006, 5 (1), 144-56.

47. Camacho, C.; Coulouris, G.; Avagyan, V.; Ma, N.; Papadopoulos, J.; Bealer, K.; Madden, T. L., BLAST+: architecture and applications. BMC Bioinformatics 2009, 10, 421.

48. Walker, D. E.; Yang, F.; Carver, J.; Joe, K.; Michels, D. A.; Yu, X. C., A modular and adaptive mass spectrometry-based platform for support of bioprocess development toward optimal host cell protein clearance. MAbs 2017, 9 (4), 654-663.

49. Schenauer, M. R.; Flynn, G. C.; Goetze, A. M., Identification and quantification of host cell protein impurities in biotherapeutics using mass spectrometry. Anal Biochem 2012, 428 (2), 150-7.

50. Reiter, K.; Suzuki, M.; Olano, L. R.; Narum, D. L., Host cell protein quantification of an optimized purification method by mass spectrometry. J Pharm Biomed Anal 2019, 174, 650-654.

51. Picotti, P.; Aebersold, R., Selected reaction monitoring-based proteomics: workflows, potential, pitfalls and future directions. Nat Methods 2012, 9 (6), 555-66.

52. Gallien, S.; Duriez, E.; Crone, C.; Kellmann, M.; Moehring, T.; Domon, B., Targeted proteomic quantification on quadrupole-orbitrap mass spectrometer. Mol Cell Proteomics 2012, 11 (12), 170923. 
53. Peterson, A. C.; Russell, J. D.; Bailey, D. J.; Westphall, M. S.; Coon, J. J., Parallel reaction monitoring for high resolution and high mass accuracy quantitative, targeted proteomics. Mol Cell Proteomics 2012, 11 (11), 1475-88.

54. Borras, E.; Sabido, E., What is targeted proteomics? A concise revision of targeted acquisition and targeted data analysis in mass spectrometry. Proteomics 2017, 17 (17-18). 
Figure legends

Figure 1: Experimental design for evaluating sample preparation protocols and data analysis workflows for the monitoring and the accurate quantification of host cell proteins (HCP). Four commercial Drug Products (adalimumab, bevacizumab, nivolumab and trastuzumab) were prepared in triplicate according to four different protocols: a native digestion (ND), an optimized liquid digestion (OLD), an optimized native digestion (OND) and a semi-fractionation protocol. After retention time standards (iRT peptides) and standard proteins (ADH, PYGM, BSA and ENL) spike-in, samples were analyzed in both DDA and DIA modes on a nanoLC-Q-Exactive HF-X system. DDA data was processed with Proteome Discoverer and DIA data with directDIA, while applying stringent validation criteria. Finally, a Top3 strategy was used to obtain HCP quantity estimations.

Figure 2: Effects of the validation filters on the number of HCP peptides and proteins in the case of optimized liquid digestion (OLD) protocol.

Number of quantified HCP peptides (A) and protein groups (B) for the different mAb samples after sequentially applying validation filters - no filters (None, i.e. preliminary filters only), signal quality filter, signal repeatability filter, and homology filter - on the DDA (left) and DIA (right) data. Filters are described in the results and discussion section.

Figure 3: Comparison of sample preparation protocols for HCP monitoring and accurate quantification.

A-B. Number of quantified HCP peptides (A) and protein groups (B) for the different mAb samples analysed in DDA (left) and DIA (right). 
C. Global HCP amount obtained for the different mAb samples analyzed in DDA (left) and DIA (right). Bar height represents the mean of the technical triplicate, and error bars the standard deviation.

OND: Optimized native digestion protocol. OLD: Optimized liquid digestion protocol. ND: Native digestion protocol.

Figure 4: HCP quantification performances depending on the sample preparation protocol and the acquisition mode.

Boxplots representing the distribution of the PYGM/ADH MS2 signal estimated on the mean of each mAb technical triplicate (A), and the distribution of the coefficients of variation (CV) on HCP precursor signal in each replicate of each mAb sample (B), prepared with the native digestion (ND), optimized liquid digestion (OLD) or optimized native digestion (OND) protocol, and analyzed in DDA (light grey) or DIA (orange) mode. In A, the black dotted line corresponds to the expected ratio value of 0.2. In $\mathrm{A}$ and $\mathrm{B}, \mathrm{N}=4$, except for ND-DDA, where $\mathrm{N}=3$. 
Figure 1

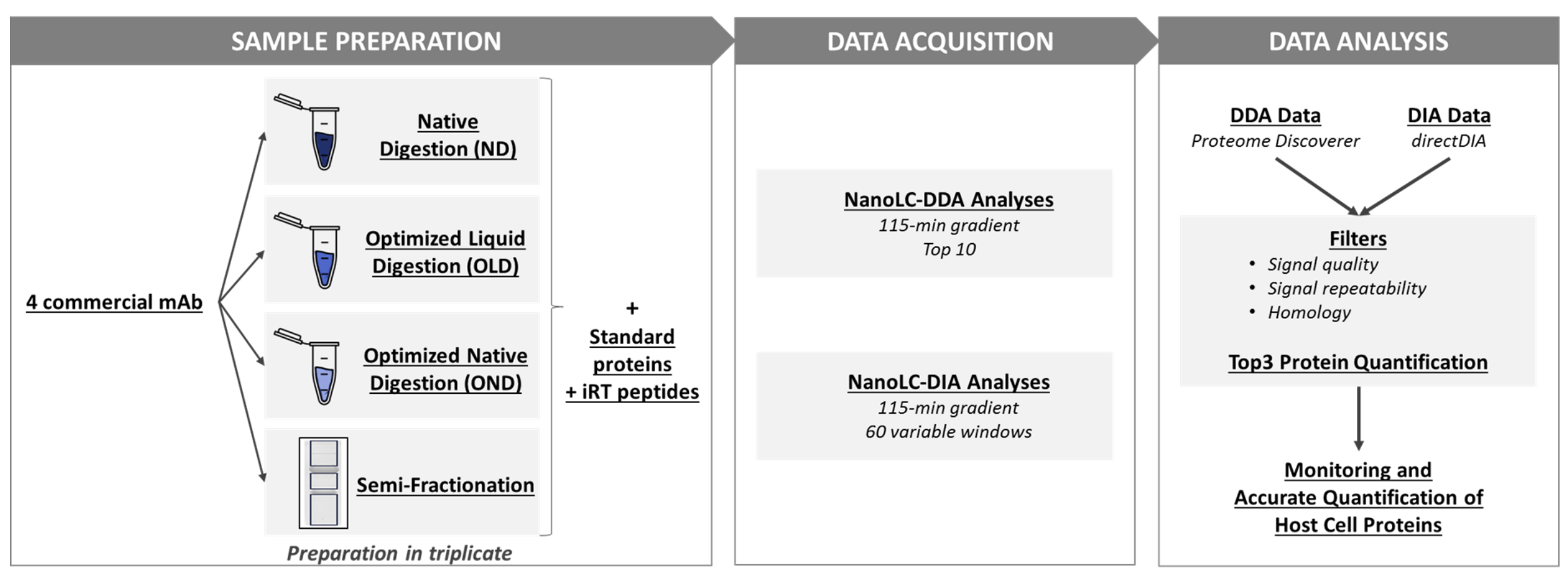


Figure 2
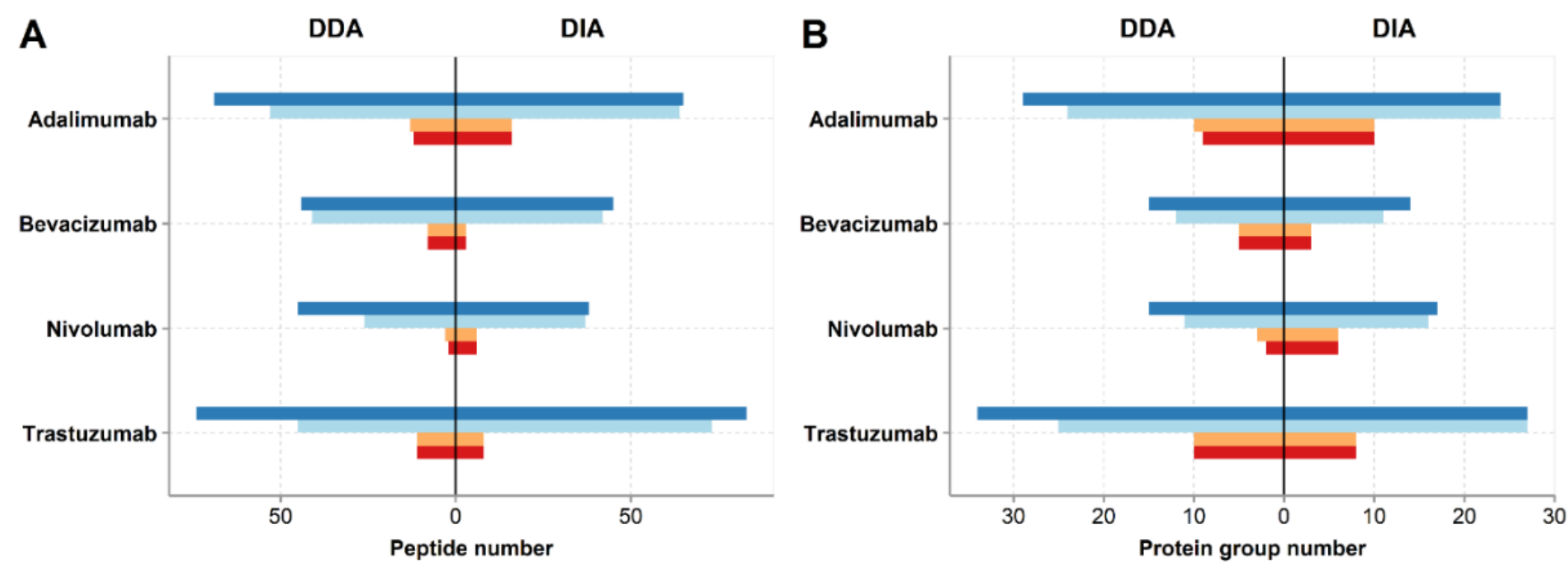

None Signal quality

Signal repeatability

Homology 
Figure 3
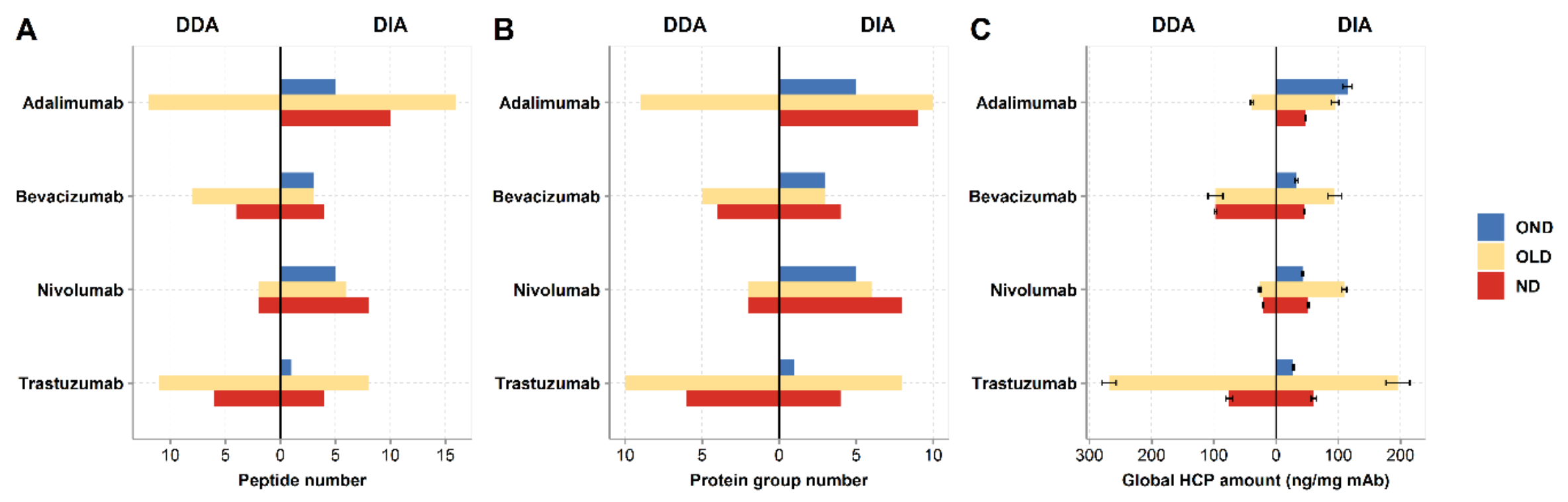
Figure 4

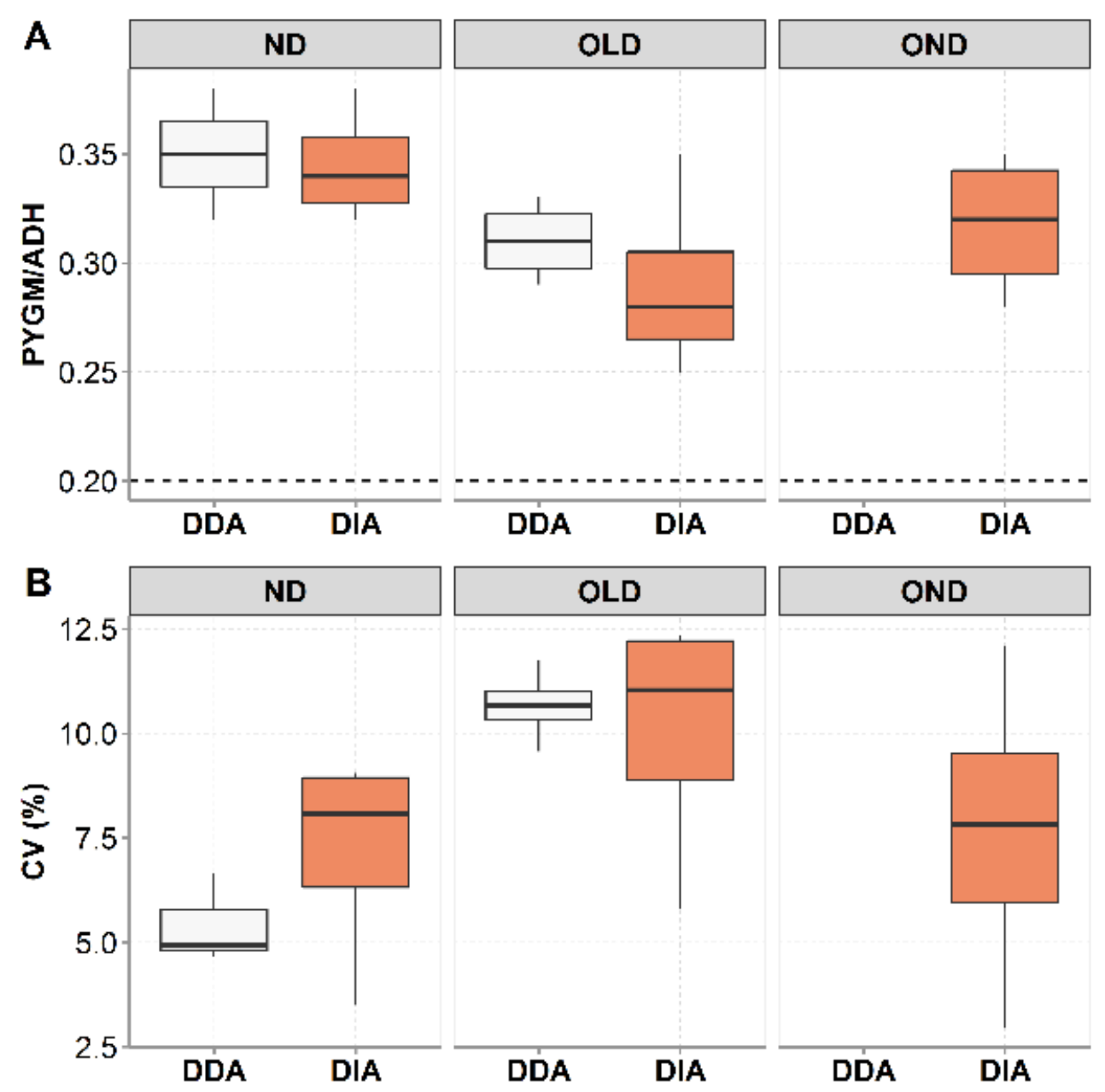


For TOC only

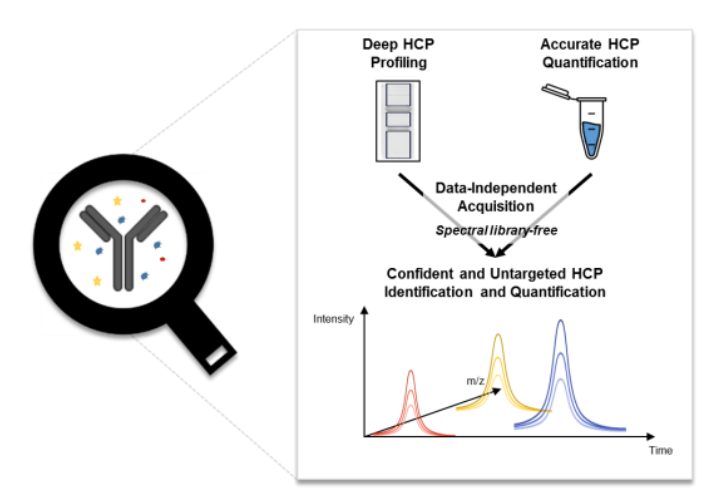

